\title{
Benefit of volumetric-modulated arc therapy over three- dimensional conformal radiotherapy for stage I-II extranodal marginal zone B-cell lymphoma of mucosa-associated lymphoid tissue in the stomach: a dosimetric comparison
}

\author{
Joo-Hyun Chung, MD', Kyoungsu Na, BS, RTT', II Han Kim, MD, PhD ${ }^{1,2}$ \\ 'Department of Radiation Oncology, Seoul National University Hospital, Seoul National University College of Medicine, Seoul; \\ ${ }^{2}$ Cancer Research Institute, Seoul National University, Seoul, Korea
}

Purpose: To retrospectively analyze dosimetric parameters of volumetric-modulated arc therapy (VMAT) and three-dimensional conformal radiotherapy (3D-CRT) delivered to extranodal marginal zone B-cell lymphomas of mucosa-associated lymphoid tissue in the stomach (gastric MALT lymphoma) to find out advantages of VMAT and conditions for definite benefits of VMAT.

Materials and Methods: Fifty patients with stage I-II gastric MALT Iymphoma received VMAT $(n=14)$ or 3D-CRT ( $n=36)$ between December 2005 and April 2018. Twenty-seven patients were categorized according to whether the planning target volume (PTV) overlaps kidney(s). Dosimetric parameters were analyzed by dose-volume histogram.

Results: Radiation dose to the liver was definitely lower with VMAT in terms of mean dose $(p=0.026)$ and $V_{15}(p=0.008)$. The $V_{15}$ of the left kidney was lower with VMAT $(p=0.065)$. For those with PTV overlapping kidney $(s)$, the left kidney $V_{15}$ was significantly lower with VMAT. Furthermore, the closer the distance between the PTV and kidneys, the less the left kidney $V_{15}$ with VMAT $(p=0.037)$. Delineation of kidney(s) by integrating all respiratory phases had no additional benefit.

Conclusions: VMAT significantly increased monitor units, reduced treatment time and radiation dose to the liver and kidneys. The benefit of VMAT was definite in reducing the left kidney $V_{15}$ especially in geometrically challenging conditions of overlap or close separation between PTV and kidney(s).

Keywords: Volumetric-modulated arc therapy, 3-D conformal radiotherapy, Gastric MALT lymphoma, Organs at risk, Kidney dose, Liver dose

\section{Introduction}

Extranodal marginal zone B-cell lymphoma of mucosaassociated lymphoid tissue (MALT lymphoma) arises in the stomach, ocular adnexa, salivary gland, lung, small bowel, thyroid, and skin. The most common gastric MALT Iymphoma, diffuse or multifocal [1,2], is often associated with Helicobacter pylori (HP) in its pathogenesis. The first-line treatment of gastric MALT Iymphoma is antibiotic therapy if HP has been identified. However, radiotherapy becomes the treatment of choice in the absence of HP or when the eradication of HP has failed $[3,4]$. Radiotherapy alone yields excellent local tumor

Received 28 August 2018, Revised 29 October 2018, Accepted 27 November 2018.

Correspondence: II Han Kim, MD, PhD, Department of Radiation Oncology, Seoul National University Hospital, Seoul National University College of Medicine, 101 Daehak-ro, Jongno-gu, Seoul 03080, Korea. Tel: +82-2-2072-2528, Fax: +82-2765-3317, E-mail: ihkim@snu.ac.kr (http://orcid.org/0000-0002-4755-5201)

(c) This is an Open Access article distributed under the terms of the Creative Commons Attribution Non-Commercial License (http://creativecommons.org/ licenses/by-nc/4.0/) which permits unrestricted non-commercial use, distribution, and reproduction in any medium, provided the original work is properly cited.

www.e-roj.org 
control rate of 95\% or more as well as long-term survival [5-9].

During radiotherapy, the radiation field encompasses whole stomach only. Perigastric lymph nodes are added only if their involvement is clinically confirmed $[10,11]$. Due to intra-fractional movement of the stomach and the very closely located organs at risk (OARs) such as kidney and liver, it is difficult to decide adequate planning target volume (PTV) margins and achieve optimal planning to avoid OARs. Previous studies have reported that intensity-modulated radiation therapy (IMRT) has advantages in reducing OAR doses compared to other planning methods including threedimensional conformal radiotherapy (3D-CRT) [12-16]. However, there is some evidence that IMRT generates higher scattered doses due to its longer beam-on time and increased collimator scatter [17]. However, no precedent studies have compared volumetric-modulated arc therapy (VMAT) with other planning methods. Therefore, the objective of this retrospective study was to elucidate possible advantages of VMAT by comparing dosimetric parameters between VMAT and 3D-CRT for gastric MALT Iymphoma. In addition, we tried to find out conditions that could have definite benefit of VMAT.

\section{Materials and Methods}

\section{Patients}

A total of 71 gastric MALT Iymphoma patients received radiotherapy at Seoul National University Hospital between December 2005 and April 2018. All patients were pathologically confirmed with gastric MALT Iymphoma by endoscopy. Radiotherapy was not given to any patient previously. Twentyone patients were excluded from this study because they did not have assessable dose-volume histogram (DVH). Clinical data, radiotherapy plan, and DVH of the remaining 50 patients were retrospectively reviewed after obtaining approval from the Institutional Review Board of Seoul National University Hospital (No. H-1804-135-940).

\section{Radiotherapy techniques}

Radiotherapy method was 3D-CRT based on conventional computed tomography (CT) until September 2017. It was VMAT using four-dimensional computed tomography (4D-CT) thereafter. Ingesting $50 \mathrm{~mL}$ of barium after overnight fasting, patients underwent fluoroscopy just before CT simulation to assess direction and magnitude of real-time movement of the stomach. Diaphragmatic and stomach movements were recorded to determine optimal PTV margins. They were used as reference for weekly check. CT images were then reconstructed with slice thickness of $3 \mathrm{~mm}$ for patients at supine position secured with an arm-up holder.

For 3D-CRT, clinical target volume (CTV) was defined as the whole stomach and involved regional lymph nodes. Individual PTV were delineated by each patient's stomach movement. It usually encompassed CTV plus $1.5-2.5 \mathrm{~cm}$ margin in the craniocaudal direction and $0.5-1.0 \mathrm{~cm}$ in the radial direction. For VMAT, internal target volume (ITV) was created by summating every CTV at each respiratory phase after 4D-CT. PTV was defined as ITV plus $0.5 \mathrm{~cm}$ in all directions.

OARs such as right and left kidneys, liver, right and left lungs, duodenum, and spinal cord were contoured. For VMAT plan, ITV and OARs contoured in 50\% phase of respiration were used for DVH analysis. To compensate interplay effect between stomach and kidney, planning organ at risk volume (PRV) of both kidneys was generated by summating all kidney structures from every respiratory phase. Such generated kidney structure was designated as integrated-PRV (iPRV). Eclipse treatment planning system (version 13.6; Varian Medical Systems, Palo Alto, CA, USA) was used to plan radiotherapy.

For 3D-CRT plans, the number of coplanar or non-coplanar fields ranged from 3 to 6 , with median of 5 fields. Noncoplanar beams were usually inferior oblique beams to save the left kidney. VMAT plans consisted of two fields, avoiding arc angles facing OARs as much as possible. Optimal conformity index, homogeneity index, and dose limits of OARs were achieved by optimizing beam angles, weights, and multi-leaf collimators. Dose reduction of the left kidney and liver was achieved as much as possible without compromising PTV coverage. These plans were normalized so that at least 95\% of the PTV received prescribed dose.

The daily dose was $1.8 \mathrm{~Gy}$ in a single fraction. The total dose was $30.6 \mathrm{~Gy}$ in 38 patients and <30.6 Gy in 12 patients. For unbiased comparison of dose to OARs, total doses were standardized to 30.6 Gy. Geometric relationship between PTV and kidneys of the patients was categorized into type I or II (Fig. 1) which was simplified from a three-tiered categorization [12]

\section{Dosimetric parameters for plan evaluation}

Dose distribution for each plan was analyzed using DVHs in the Eclipse system. OAR doses were evaluated using the following criteria: (1) mean dose and percent volume of each kidney receiving 15 Gy or more $\left(\mathrm{V}_{15}\right)$; (2) mean dose and $\mathrm{V}_{15}$ of liver and duodenum. $V_{15}$ was chosen because half dose of 30.6 Gy represents the median value, which is most appropriate in evaluating organs at risk. PTV doses were evaluated using the following three parameters to evaluate target coverage: 
A

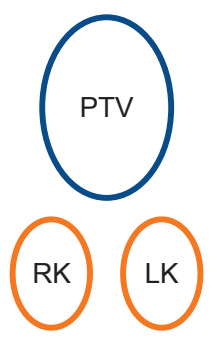

AP view

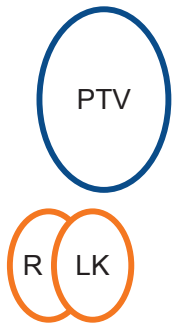

Lateral view
B

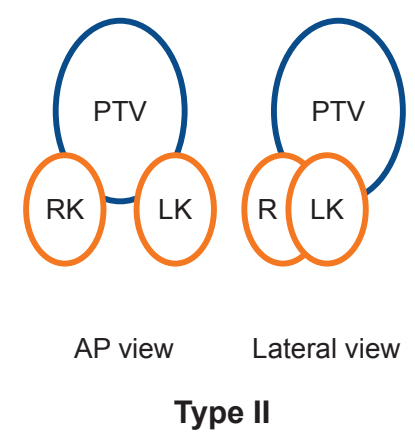

Fig. 1. Categorization of geometric relationship of the PTV and kidney(s) according to no overlap between PTV and kidney(s) (type I, A) and overlap between PTV and kidney(s) (type II, B). PTV, planning target volume; RK, right kidney; LK, left kidney

(1) percent volume of PTV receiving at least 95\% of the prescription dose $\left(\mathrm{PTV}_{95}\right) ;(2)$ the minimum dose received by $95 \%$ of the PTV $\left(D_{95}\right) ;(3)$ the minimum dose received by $5 \%$ of the PTV $\left(D_{5}\right)$. The conformity index $(C l)$ was calculated as follows:

$$
C l=\frac{P T V_{95}}{P T V_{\text {total }}}
$$

where PTV $_{95}$ was the volume of PTV receiving 95\% of the prescribed dose and PTV total $_{\text {was }}$ the total volume of PTV [18]. A higher value indicates a better dose coverage and an ideal $\mathrm{Cl}$ is 1. Homogeneity index (HI) was calculated as follows:

$$
H I=\frac{D_{5}-D_{95}}{D_{50}}
$$

where $D_{5}$ and $D_{95}$ were the minimum dose in $5 \%$ and $95 \%$ of the PTV, respectively, and $D_{50}$ was the minimum dose in $50 \%$ of the PTV [19]. A lower value indicates a more homogeneous dose distribution and ideal $\mathrm{HI}$ is 0 . Mean doses of the right and left kidney, liver, and duodenum as well as $V_{15}$ were calculated from DVH.

\section{Statistical analysis}

The 3D-CRT and VMAT parameters were compared using Student t-test. Mann-Whitney U-test was used during subgroup analysis on type I patients due to a small number of patients. A p-value less than 0.05 was considered statistically significant. All statistical analyses were performed using STATA software version 14.0 (StataCorp., College Station, TX, USA).

\section{Results}

\section{Patient characteristics}

Clinical features of 50 patients are summarized in Table 1. Thirty-six patients received 3D-CRT while 14 patients received VMAT. Their median age was 53 years (range, 25 to 80 years). There were 21 males and 29 females. Twenty-one patients (42.0\%) were HP positive while 29 (58.0\%) were HP negative. Forty-one patients (82.0\%) had clinical stage IE while 9 (18.0\%) had stage IIE.

Mean PTV margin and median PTV volume were significantly lower in the VMAT group than those in the 3D-CRT group. The median PTV volume was $575.1 \mathrm{~mL}$ (range, 334.0 to $1,362.0 \mathrm{~mL}$ ) in all, $685.4 \mathrm{~mL}$ (range, 334.0 to $1,362.0$ $\mathrm{mL}$ ) in the 3D-CRT group, and $492.9 \mathrm{~mL}$ (range, 402.3 to 611.7 $\mathrm{mL}$ ) in the VMAT group ( $p=0.010)$. The median ITV volume was $301.8 \mathrm{~mL}$ (range, 221.9 to $368.0 \mathrm{~mL}$ ) in the VMAT group. Overlapping of PTV and kidney(s) (type II) was observed in 23 (46.0\%) patients.

\section{PTV coverage and dose to OARs}

Dose-volumetric parameters are summarized in Table 2. Parameters for PTV coverage expressed as $\mathrm{D}_{\text {max }} \mathrm{V}_{98,} \mathrm{Cl}$, and $\mathrm{HI}$ were significantly better with VMAT compared to those with 3D-CRT (all $p<0.05$ ). However, $D_{\text {mean }}$ was not significantly ( $p=$ 0.444) different between the two groups.

Regarding doses to OARs, $V_{15}$ for the left kidney ( $p=0.065$ ) and $D_{\text {mean }}$ and $V_{15}$ for the liver $(p<0.05)$ were lower in the VMAT group compared to those in the 3D-CRT group. However, $D_{\text {mean }}$ of the left kidney was not lower in VMAT $(p=0.372)$. The dose to the right kidney or duodenum did not differ significantly between the two groups.

\section{Geometric relationship between PTV and kidney(s)}

1) Geometric type vs. VMAT benefit

Doses to OARs were influenced by a certain geometric condition (Table 3). Doses to all four OARs were higher in type II (overlapped group) than those in type I (non-overlap) regardless of technique. In the type I group, dosimetric benefit of VMAT over 3D CRT was marginal for the liver ( $p$ $=0.097)$. Such benefit was not observed in kidneys or the duodenum.

In type II group, VMAT was clearly superior to 3D-CRT in reducing kidney $V_{15}$ by $69 \%$ ( $p=0.007$ ). It was marginally beneficial in the liver $(p=0.089)$, but not beneficial in the right kidney or the duodenum. 
Table 1. Patients' characteristics

\begin{tabular}{|c|c|c|c|c|}
\hline & All patients & $3 \mathrm{D}-\mathrm{CRT}(\mathrm{n}=36)$ & VMAT $(n=14)$ & $p$-value \\
\hline \multicolumn{5}{|l|}{ Gender } \\
\hline Male & $21(42.0)$ & $18(50.0)$ & $3(21.4)$ & \multirow[t]{2}{*}{$0.066^{a)}$} \\
\hline Female & $29(58.0)$ & $18(50.0)$ & $11(78.6)$ & \\
\hline Age (yr) & $53(25-80)$ & $53(25-80)$ & $49(47-69)$ & $0.857^{b)}$ \\
\hline$\geq 60$ & $20(40.0)$ & $15(41.7)$ & $5(35.7)$ & $0.700^{a)}$ \\
\hline$<60$ & $30(60.0)$ & $21(58.3)$ & $9(64.3)$ & \\
\hline \multicolumn{5}{|l|}{ H. Pylori } \\
\hline Positive & $21(42.0)$ & $17(47.2)$ & $4(28.6)$ & \multirow[t]{2}{*}{$0.230^{a)}$} \\
\hline Negative & $29(58.0)$ & $19(52.8)$ & $10(71.4)$ & \\
\hline \multicolumn{5}{|l|}{ Stage } \\
\hline IE & $41(82.0)$ & $30(83.3)$ & $11(78.6)$ & \multirow[t]{2}{*}{$0.514^{a)}$} \\
\hline IIE & $9(18.0)$ & $6(16.7)$ & $3(21.4)$ & \\
\hline PTV volume (mL) & $575.1(334.0-1,362.0)$ & $685.4(334.0-1,362.0)$ & $492.9(402.3-611.7)$ & $0.010^{b)}$ \\
\hline$\geq 600$ & $21(42.0)$ & $20(55.6)$ & $1(7.1)$ & \multirow[t]{2}{*}{$0.002^{a)}$} \\
\hline$<600$ & $29(58.0)$ & $16(44.4)$ & $13(92.9)$ & \\
\hline Mean PTV margin (cm) & $1.06 \pm 0.62$ & $1.28 \pm 0.60$ & $0.50 \pm 0.00$ & $<0.001^{b)}$ \\
\hline \multicolumn{5}{|c|}{ PTV overlap with kidney(s) } \\
\hline No (type I) & $27(54.0)$ & $17(47.2)$ & $10(71.4)$ & \multirow[t]{2}{*}{$0.123^{\mathrm{a})}$} \\
\hline Yes (type II) & $23(46.0)$ & $19(52.8)$ & $4(28.6)$ & \\
\hline
\end{tabular}

Values are presented as number (\%) or median (range) or mean \pm standard deviation. 3D-CRT, three-dimensional conformal radiotherapy; VMAT, volumetric modulated arc therapy; HP, Helicobacter pylori; PTV, planning target volume.

${ }^{\text {a) }}$ Fisher exact test, ${ }^{\text {b) }}$ Student t-test.

Table 2. Dose-volume parameters of PTV and OARs by 3D-CRT or VMAT plan

\begin{tabular}{|c|c|c|c|}
\hline Parameter & $3 \mathrm{D}-\mathrm{CRT}(\mathrm{n}=36)$ & VMAT $(n=14)$ & $p$-value ${ }^{a)}$ \\
\hline \multicolumn{4}{|l|}{ PTV } \\
\hline $\mathrm{D}_{\max }(\mathrm{Gy})$ & $32.7 \pm 0.5$ & $33.4 \pm 0.3$ & $<0.005$ \\
\hline$D_{\text {mean }}(G y)$ & $30.6 \pm 4.3$ & $31.5 \pm 0.1$ & 0.444 \\
\hline$V_{98}(\%)$ & $97.0 \pm 3.8$ & $99.7 \pm 0.5$ & 0.010 \\
\hline Conformity index & $0.970 \pm 0.038$ & $0.997 \pm 0.005$ & 0.010 \\
\hline Homogeneity index & $0.056 \pm 0.011$ & $0.042 \pm 0.009$ & $<0.001$ \\
\hline \multicolumn{4}{|l|}{ Right kidney } \\
\hline$D_{\text {mean }}(G y)$ & $3.1 \pm 2.7$ & $4.7 \pm 2.3$ & 0.096 \\
\hline$V_{15}(\%)$ & $5.6 \pm 9.1$ & $3.8 \pm 5.9$ & 0.473 \\
\hline \multicolumn{4}{|l|}{ Left kidney } \\
\hline$D_{\text {mean }}(G y)$ & $5.7 \pm 4.3$ & $4.8 \pm 3.2$ & 0.372 \\
\hline$V_{15}(\%)$ & $15.1 \pm 16.1$ & $6.7 \pm 9.9$ & 0.065 \\
\hline \multicolumn{4}{|l|}{ Liver } \\
\hline$D_{\text {mean }}(G y)$ & $10.5 \pm 3.8$ & $8.0 \pm 1.7$ & 0.026 \\
\hline$V_{15}(\%)$ & $28.3 \pm 16.4$ & $16.3 \pm 3.4$ & 0.008 \\
\hline \multicolumn{4}{|l|}{ Duodenum } \\
\hline$D_{\text {mean }}(G y)$ & $13.1 \pm 7.8$ & $15.9 \pm 6.9$ & 0.259 \\
\hline$V_{15}(\%)$ & $39.9 \pm 28.3$ & $48.6 \pm 24.6$ & 0.201 \\
\hline
\end{tabular}

Values are presented as mean \pm standard deviation.

PTV, planning target volume; OAR, organs at risk; 3D-CRT, three-dimensional conformal radiotherapy; VMAT, volumetric modulated arc therapy; $D_{\text {maxı }}$ maximum dose; $D_{\text {mean }}$ mean dose adjusted to the total dose of $30.6 \mathrm{~Gy} ; V_{98}$, percentage of the PTV receiving $98 \%$ of the prescription dose; $V_{15}$, percentage of irradiated volume receiving $15 \mathrm{~Gy}$ or higher.

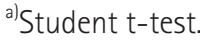


Table 3. Dose-volume parameters of OAR by radiotherapy technique and the status of PTV overlap with kidney(s)

\begin{tabular}{|c|c|c|c|c|c|}
\hline & & $\begin{array}{l}\text { PTV overlap with } \\
\text { kidney(s) }\end{array}$ & 3D-CRT & VMAT & $p$-value ${ }^{a)}$ \\
\hline \multirow[t]{4}{*}{$V_{15}(\%)$} & Right kidney & No & $4.42 \pm 9.58(n=17)$ & $3.67 \pm 5.52(n=10)$ & 0.728 \\
\hline & & Yes & $6.62 \pm 8.84(n=19)$ & $4.11 \pm 7.71(n=4)$ & 0.407 \\
\hline & Left kidney & No & $6.68 \pm 13.00(n=17)$ & $6.64 \pm 11.70(n=10)$ & 0.306 \\
\hline & & Yes & $22.60 \pm 15.10(n=19)$ & $6.95 \pm 3.86(n=4)$ & 0.007 \\
\hline \multirow{4}{*}{$D_{\text {mean }}(G y)$} & Liver & No & $9.2 \pm 2.0(n=17)$ & $7.9 \pm 1.9(n=10)$ & 0.097 \\
\hline & & Yes & $11.6 \pm 4.7(n=19)$ & $8.3 \pm 1.1(n=4)$ & 0.089 \\
\hline & Duodenum & No & $13.1 \pm 7.5(n=17)$ & $15.4 \pm 5.9(n=10)$ & 0.246 \\
\hline & & Yes & $13.2 \pm 8.4(n=19)$ & $17.2 \pm 10.7(n=4)$ & 0.395 \\
\hline
\end{tabular}

Values are presented as mean \pm standard deviation.

PTV, planning target volume; OAR, organs at risk; 3D-CRT, three-dimensional conformal radiotherapy; VMAT, volumetric modulated arc therapy; $V_{15}$, percentage of irradiated volume receiving $15 \mathrm{~Gy}$ or higher.

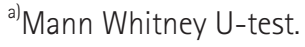

\section{2) Distance between PTV and kidney(s) vs. VMAT benefit} Minimal distances between the PTV and both right and left kidneys were measured using the Eclipse treatment planning system. Mean minimal distances from the PTV to the right kidney and the left kidney were $3.45 \pm 2.23 \mathrm{~cm}$ and $1.46 \pm$ $0.78 \mathrm{~cm}$, respectively. Scatter plots based on the relationship between the minimal distance from the PTV to kidney and V15 of the right and left kidneys are constructed. A quadratic

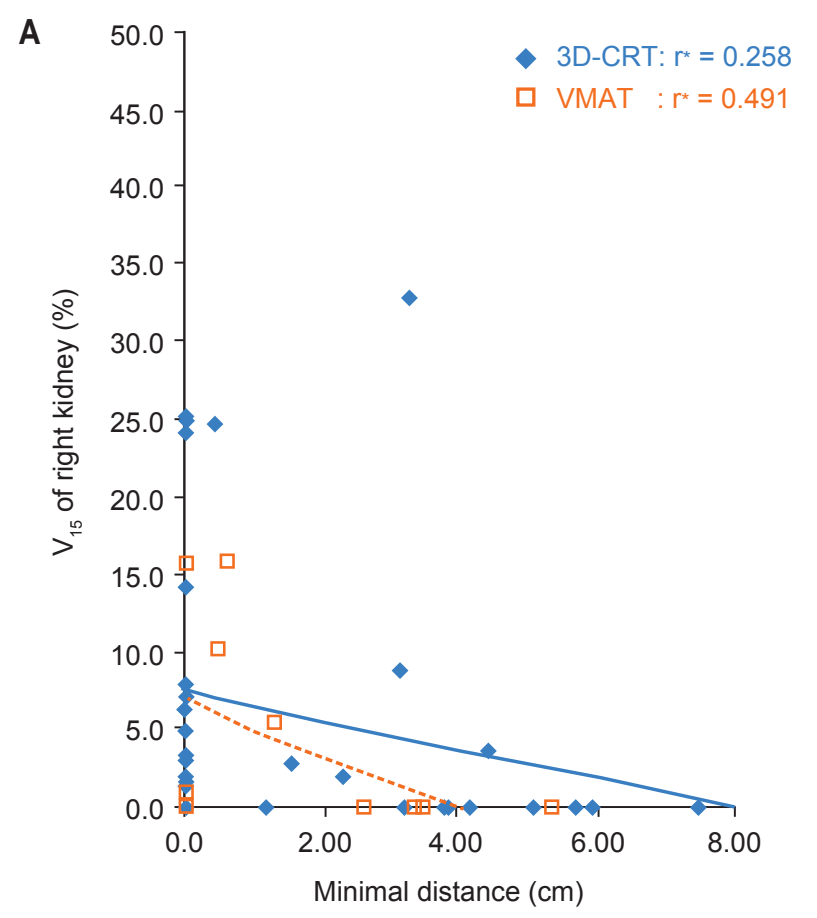

regression line was plotted and Pearson correlation coefficient was calculated (Fig. 2). The Pearson correlation coefficient was significant only for the 3D-CRT group in the left kidney $(r=$ -0.477, $p=0.003$ ). However, the overall trend showed that the difference between $\mathrm{V}_{15}$ of 3D-CRT and VMAT in the right kidney was decreased when the distance was decreased whereas the difference between $V_{15}$ of 3D-CRT and VMAT in the left kidney was increased as the distance was decreased. When the

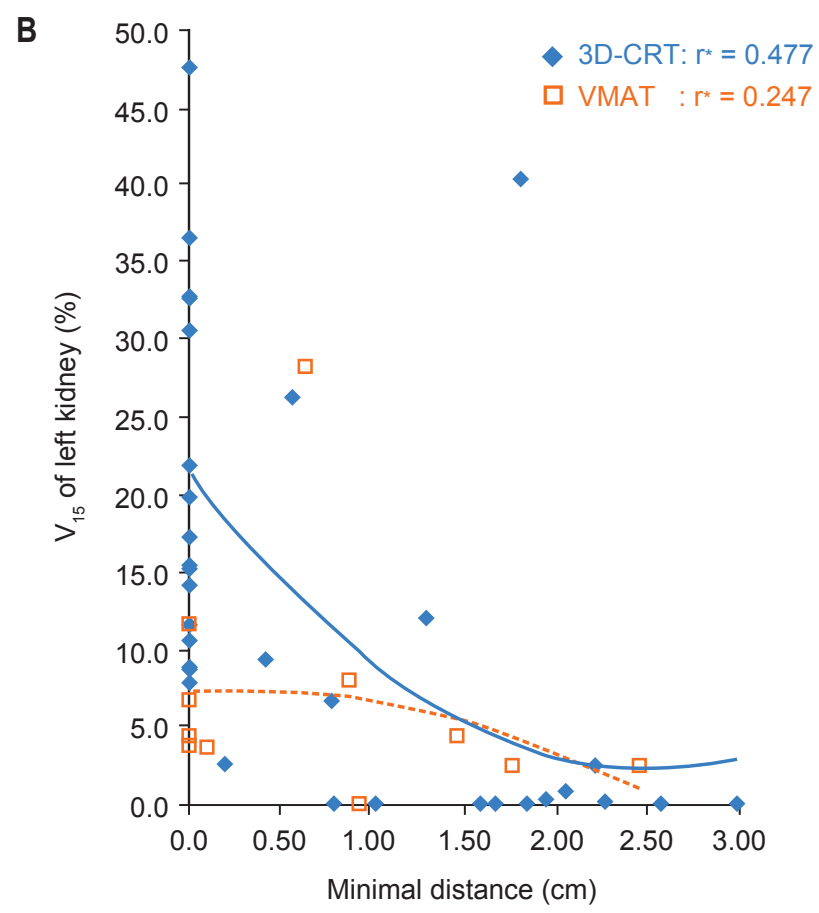

Fig. 2. Scatter plot of the kidney $V_{15}$ by its minimal distance from the PTV in the right kidney (A) and the left kidney (B). *Pearson correlation coefficient. 
distance was less than $2 \mathrm{~cm}$, VMAT had significantly ( $p=0.037)$ lower $V_{15}$ of the left kidney (Table 4).

3) Comparison between VMAT and iPRV-corrected VMAT plans When the ITV concept was applied to the target volume, static images at the mid-expiratory phase was chosen to represent OARs. Thus, there might be an error or inaccuracy in assessment of the dose to OARs defined in a single respiratory phase.

After integrating PRVs of the kidneys of all respiratory phases into iPRV, dose-volume parameters of simple VMAT plan and iPRV-corrected VMAT plan were compared. Although
PTV volumes for both kidneys were obviously larger in iPRVcorrected VMAT plan, neither mean doses nor $V_{15}$ of both kidneys differed significantly (Table 5).

\section{Treatment time and monitor units}

Daily treatment times from beam-on to beam-off were measured at the 8th and 9th sessions of radiotherapy for all patients. Time consumed for immobilization or set-up was excluded from rational comparison. With VMAT, beam delivery time was reduced to about $1 / 3$ of the time required for 3D-CRT while monitor unit was increased by a factor of 1.5 (Table 6).

Table 4. $V_{15}$ of the kidney by the minimal distance between PTV and non-overlapped kidney(s)

\begin{tabular}{rcccc}
\hline$V_{15}(\%)$ & Minimal distance $(\mathrm{cm})$ & 3D-CRT & VMAT & $p$-value $e^{\mathrm{a})}$ \\
\hline Right kidney & $\leq 2.0$ & $6.98 \pm 9.23(\mathrm{n}=22)$ & $6.65 \pm 6.57(\mathrm{n}=8)$ & 0.739 \\
& $>2.0$ & $3.39 \pm 8.85(\mathrm{n}=14)$ & $0.00 \pm 0.00(\mathrm{n}=6)$ & 0.157 \\
Left kidney & $\leq 2.0$ & $16.90 \pm 16.20(\mathrm{n}=32)$ & $7.43 \pm 10.60(\mathrm{n}=12)$ & 0.037 \\
& $>2.0$ & $0.75 \pm 1.40(\mathrm{n}=4)$ & $2.57 \pm 0.00(\mathrm{n}=2)$ & 0.340
\end{tabular}

Values are presented as mean \pm standard deviation.

PTV, planning target volume; 3D-CRT, three-dimensional conformal radiotherapy; VMAT, volumetric modulated arc therapy; $V_{15}$, percentage of irradiated volume receiving 15 Gy or higher.

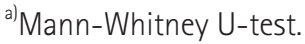

Table 5. Dose-volume parameters of kidneys: VMAT plan vs. iPRV-corrected VMAT plan

\begin{tabular}{lccc}
\hline \multicolumn{1}{c}{ Parameter } & VMAT & iPRV-corrected VMAT & p-value ${ }^{\text {a) }}$ \\
\hline Right kidney & & & \\
Volume $(\mathrm{mL})$ & $128.3 \pm 16.7$ & $162.2 \pm 13.1$ & 0.001 \\
D $_{\text {mean }}(\mathrm{Gy})$ & $4.7 \pm 2.3$ & $4.6 \pm 2.3$ & 0.893 \\
V $_{15}(\%)$ & $3.8 \pm 5.9$ & $3.8 \pm 5.7$ & 0.634 \\
Left kidney & & & $<0.001$ \\
Volume $(\mathrm{mL})$ & $135.4 \pm 19.2$ & $177.2 \pm 30.7$ & 0.888 \\
$D_{\text {mean }}(\mathrm{Gy})$ & $4.8 \pm 3.2$ & $4.7 \pm 3.2$ & 0.682 \\
$V_{15}(\%)$ & $6.7 \pm 9.9$ & $5.8 \pm 7.3$ &
\end{tabular}

Values are presented as mean \pm standard deviation.

VMAT, volumetric modulated arc therapy; iPRV, integrated planning organ at risk volume; $D_{\text {mean }}$ mean dose; $V_{15}$, percentage of irradiated volume receiving $15 \mathrm{~Gy}$ or higher.

a) Student t-test.

Table 6. Differences in treatment time and monitor unit by radiotherapy technique

\begin{tabular}{lccc}
\hline & 3D-CRT $(n=36)$ & VMAT $(n=14)$ & $p$-value \\
\hline Treatment time $(\min )^{\text {b) }}$ & $5.6 \pm 1.1$ & $1.6 \pm 0.6$ & $<0.001$ \\
Monitor unit & $235.6 \pm 20.5$ & $366.6 \pm 23.6$ & $<0.001$ \\
\hline
\end{tabular}

Values are presented as mean \pm standard deviation.

3D-CRT, three-dimensional conformal radiotherapy; VMAT, volumetric modulated arc therapy.

a) Student t-test. ${ }^{\text {b) }}$ From beam-on to beam-off, not including time consumed for set-up or immobilization. 


\section{Discussion and Conclusion}

This study revealed that the VMAT plan was superior to the 3D-CRT plan in both PTV coverage and normal organ sparing in radiotherapy for gastric MALT Iymphoma. In particular, VMAT was an effective tool for reducing the left kidney dose in a geometrically challenging patient, in which PTV and kidneys were overlapping or closely located.

Both $\mathrm{Cl}$ and $\mathrm{HI}$ were significantly better with the VMAT plan compared to those with a 3D-CRT plan, although the difference was small. Various studies have revealed that IMRT plan yields better value in $\mathrm{Cl}$ and $\mathrm{HI}$ than 3D-CRT $[14,20]$, although other studies have reported that there is no significant difference between the two $[12,15]$. Most precedent studies used step-and-shoot IMRT rather than VMAT which was utilized in the present study. Adorante et al. [21] have reported that VMAT in upper abdominal tumors is superior to step-and-shoot IMRT regarding $\mathrm{Cl}$. Because homogeneous dose distribution can be achieved by constantly rotating arc in VMAT, one may safely assert that VMAT is superior to 3D-CRT with better $\mathrm{Cl}$ and $\mathrm{HI}$.

Mean liver dose, $V_{15}$ of the liver, and $V_{15}$ of the left kidney were lower in VMAT plan. Studies on liver sparing effect in radiotherapy for hepatocellular carcinoma have reported that high dose region is smaller in IMRT while low dose region is smaller in 3D-CRT $[22,23]$. In the 3D-CRT plan, liver dose increases due to the entrance and exit dose from lateral and oblique beams through the structure which is expected. The kidney is radiosensitive. The left kidney is usually abutted or very near to the body of the stomach. Therefore, reducing radiation dose to the left kidney is the most imperative in treating gastric MALT Iymphoma patients to prevent renal toxicity.

While VMAT spared the left kidney $V_{15}$ with marginal significance, the dose-sparing effect was maximized in type II patients (PTV overlapped with kidneys). A similar study done by Della Biancia et al. [12] has shown that 3D-CRT is superior to anterior-posterior/posterior-anterior (AP/PA) parallel-opposed fields, especially for patients with abutting or overlapping PTV and kidneys. This result is probably due to abrupt falloff of dose distribution in the VMAT plan as the priority of VMAT planning is to reduce kidney dose. The relationship between PTV and the left kidney varies depending on the volume of the stomach, body mass index (BMI) of the patient, and normal breathing motion. Given that every patient performed fasting before simulation and treatment, factors that dichotomized patients into type I and II might depend on BMI and breathing motion. Therefore, after CT simulation was done and anatomies of the patient were reviewed, geometrically challenging patients such as type II patients might be selected as VMAT plan might be the most beneficial for these patients for sparing the left kidney dose. The left kidney-sparing effect remained effective as long as the minimal distance between the PTV and kidneys was less than $2 \mathrm{~cm}$. This result implies that the VMAT plan is more efficient for saving the left kidney when the kidney is located closer to the stomach PTV.

The iPRV-corrected VMAT plan was generated in order to compensate for internal organ movement during radiation. However, our result showed that the mean dose and $V_{15}$ of both kidneys remained almost the same even if volumes for both kidneys were increased when iPRV-correction was performed. Therefore, dose comparison in VMAT plans may not need iPRV-corrections for normal organs in consideration when analyzing DVH. In addition, when considering internal motion, the actual mean dose irradiated to the kidneys is less than the dose calculated on the original VMAT plan.

Such a low dose as 30.6 Gy suffices in controlling gastric MALT Iymphoma, leading to a high complete remission (CR) rate. Moreover, because studies trying to reduce the dose as low as 27 Gy or less is ongoing, normal organ toxicity may become a less critical issue when treating gastric MALT lymphoma. However, whose stomach is located too close to the neighboring kidney (especially the left kidney), some patients may suffer kidney toxicity issues. Some studies have reported late renal toxicity in long-term follow-up studies of patients undergoing total-body irradiation even when the dose is under $15 \mathrm{~Gy}[24,25]$. Therefore, the VMAT plan is responsible for the main improvement in the left kidney dose. However there might be potential late radiation effect from increased integral dose due to significantly increased monitor units with VMAT.

This study has some limitations. First, this was a retrospective study comparing 50 patients treated at different time points. No additional plans were generated for a single patient. Therefore, difference in patient characteristics according to treatment modality exists. The PTV volume was significantly different between the two treatment groups. Since PTV margins in the 3D-CRT group were compensated by the degree of motion of the stomach measured in $2 D$ simulation, median PTV margin in the 3D-CRT group was larger than that of the VMAT group. Thus, the mean PTV volume was larger in the 3D-CRT group. Such factor might have confounded the result, thus diluting the effect of difference in treatment modality itself. However, as shown in Table 5, there 
was little influence on the mean dose of both kidneys even when the volume was increased in the iPRV-corrected group. This result may be extrapolated to explain that PTV volume differences might have little influence.

In summary, VMAT can significantly reduce treatment time and radiation dose to OARs such as the liver and kidneys for gastric MALT Iymphoma. VMAT is especially effective for reducing the left kidney $V_{15}$ in geometrically challenging patients whose PTV and kidneys are overlapping or closely located.

\section{Conflict of Interest}

No potential conflic of interest relevant to this article was reported.

\section{References}

1. Harris NL, Jaffe ES, Stein $H$, et al. A revised European-American classification of lymphoid neoplasms: a proposal from the International Lymphoma Study Group. Blood 1994;84:136192.

2. Harris NL, Jaffe ES, Diebold J, et al. World Health Organization classification of neoplastic diseases of the hematopoietic and lymphoid tissues: report of the Clinical Advisory Committee meeting-Airlie House, Virginia, November 1997. J Clin Oncol 1999;17:3835-49.

3. Schechter NR, Portlock CS, Yahalom J. Treatment of mucosaassociated lymphoid tissue lymphoma of the stomach with radiation alone. J Clin Oncol 1998;16:1916-21.

4. Schechter NR, Yahalom J. Low-grade MALT Iymphoma of the stomach: a review of treatment options. Int J Radiat Oncol Biol Phys 2000;46:1093-103.

5. Koch $P$, Probst $A$, Berdel WE, et al. Treatment results in localized primary gastric lymphoma: data of patients registered within the German multicenter study (GIT NHL 02/96). J Clin Oncol 2005;23:7050-9.

6. Lin ML, Wirth A, Chao M, et al. Radiotherapy for low-grade gastric marginal zone lymphoma: a retrospective study. Intern Med J 2007;37:172-80.

7. Vrieling $C$, de Jong $D$, Boot $H$, de Boer JP, Wegman $F$, Aleman BM. Long-term results of stomach-conserving therapy in gastric MALT Iymphoma. Radiother Oncol 2008;87:405-11.

8. Goda JS, Gospodarowicz M, Pintilie M, et al. Long-term outcome in localized extranodal mucosa-associated lymphoid tissue lymphomas treated with radiotherapy. Cancer 2010;116:3815-24.
9. Wirth $A$, Gospodarowicz M, Aleman BM, et al. Long-term outcome for gastric marginal zone lymphoma treated with radiotherapy: a retrospective, multi-centre, International Extranodal Lymphoma Study Group study. Ann Oncol 2013;24:1344-51.

10. Zelenetz AD, Hoppe RT; NCCN Non-Hodgkin's Lymphoma Practice Guidelines Panel. NCCN: non-Hodgkin's Iymphoma. Cancer Control 2001;8(6 Suppl 2):102-13.

11. Yahalom J, Illidge $T$, Specht $L$, et al. Modern radiation therapy for extranodal lymphomas: field and dose guidelines from the International Lymphoma Radiation Oncology Group. Int J Radiat Oncol Biol Phys 2015;92:11-31.

12. Della Biancia $C$, Hunt M, Furhang E, Wu E, Yahalom J. Radiation treatment planning techniques for lymphoma of the stomach. Int J Radiat Oncol Biol Phys 2005;62:745-51.

13. Inaba $K$, Okamoto $H$, Wakita $A$, et al. Radiotherapy for gastric lymphoma: a planning study of 3D conformal radiotherapy the half-beam method, and intensity-modulated radiotherapy. J Radiat Res 2014;55:1141-5.

14. Bae SH, Kim DW, Kim MS, Shin MH, Park HC, Lim DH. Radiotherapy for gastric mucosa-associated lymphoid tissue lymphoma: dosimetric comparison and risk assessment of solid secondary cancer. Radiat Oncol J 2017;35:78-89.

15. Nour AA, Alaradi A, Mohamed A, Altuwaijri S, Rudat V. Intensity modulated radiotherapy of upper abdominal malignancies: dosimetric comparison with 3D conformal radiotherapy and acute toxicity. Radiat Oncol 2013;8:207.

16. Lim HW, Kim TH, Choi IJ, et al. Radiation therapy for gastric mucosa-associated lymphoid tissue lymphoma: dosevolumetric analysis and its clinical implications. Radiat Oncol J 2016;34:193-201.

17. Ruben JD, Lancaster $C M$, Jones $P$, Smith RL. A comparison of out-of-field dose and its constituent components for intensity-modulated radiation therapy versus conformal radiation therapy: implications for carcinogenesis. Int J Radiat Oncol Biol Phys 2011;81:1458-64.

18. Feuvret L, Noel G, Mazeron JJ, Bey P. Conformity index: a review. Int J Radiat Oncol Biol Phys 2006;64:333-42.

19. Kataria T, Sharma K, Subramani V, Karrthick KP, Bisht SS. Homogeneity Index: an objective tool for assessment of conformal radiation treatments. J Med Phys 2012;37:207-13.

20. Wang X, Li G, Zhang Y, et al. Single-arc volumetric-modulated arc therapy (sVMAT) as adjuvant treatment for gastric cancer: dosimetric comparisons with three-dimensional conformal radiotherapy (3D-CRT) and intensity-modulated radiotherapy (IMRT). Med Dosim 2013;38:395-400.

21. Adorante N, Perrotti F, Giancaterino S, et al. Dosimetric 
comparison of $3 \mathrm{D}$-conformal radiotherapy, intensitymodulated radiotherapy and volumetric-modulated ARC therapy for upper abdominal tumors. Phys Med 2016;32(Suppl 1):A.01.

22. Lee IJ, Seong J, Koom WS, et al. Selection of the optimal radiotherapy technique for locally advanced hepatocellular carcinoma. Jpn J Clin Oncol 2011;41:882-9.

23. Chen $D$, Wang $R$, Meng $X$, et al. A comparison of liver protection among 3-D conformal radiotherapy, intensity- modulated radiotherapy and RapidArc for hepatocellular carcinoma. Radiat Oncol 2014;9:48.

24. Kal HB, van Kempen-Harteveld ML. Renal dysfunction after total body irradiation: dose-effect relationship. Int J Radiat Oncol Biol Phys 2006;65:1228-32.

25. Cheng JC, Schultheiss TE, Wong JY. Impact of drug therapy, radiation dose, and dose rate on renal toxicity following bone marrow transplantation. Int J Radiat Oncol Biol Phys 2008;71:1436-43. 